\title{
DPYD genotype and haplotype analysis and colorectal cancer susceptibility in a case-control study from Slovakia
}

\author{
Tatiana Matáková ${ }^{1,2}$, Erika Halašová ${ }^{1,3}$, Henrieta Škovierová ${ }^{1}$, Anton Dzian ${ }^{4}$, Dušan Dobrota ${ }^{2,5}$ \\ and Mária Škereňová ${ }^{1,5}$ \\ ${ }^{1}$ Department of Molecular Medicine, Biomedical Center Martin, Jessenius Faculty of Medicine in Martin, Comenius University \\ in Bratislava, Martin, Slovakia \\ ${ }^{2}$ Department of Medical Biochemistry, Jessenius Faculty of Medicine in Martin, Comenius University in Bratislava, Martin, \\ Slovakia \\ ${ }^{3}$ Department of Medical Biology, Jessenius Faculty of Medicine in Martin, Comenius University in Bratislava, Martin, Slovakia \\ ${ }^{4}$ Clinic of Thoracic Surgery, University Hospital in Martin, Jessenius Faculty of Medicine in Martin, Comenius University in \\ Bratislava, Martin, Slovakia \\ ${ }^{5}$ Department of Clinical Biochemistry, University Hospital in Martin, Martin, Slovakia
}

\begin{abstract}
Dihydropyrimidine dehydrogenase (DPD) acts as the first-step enzyme catabolizing pyrimidines in vivo. DPYD gene mutations interfere with the breakdown of uracil and thymine. Genetic variations of DPYD can cause an enzyme deficiency state, which results in severe toxicity or other adverse side effects such as DNA damage or RNA damage caused by imbalance of the nucleotide pool. Our case-control study investigates the possible association between seven DPYD gene polymophisms (rs1801267, rs72547602, rs1801160, rs3918290, rs1801159, rs1801158, rs1801265) and risk of colorectal cancer (CRC). The association analysis for DPD was performed on 273 CRC patients and 187 healthy controls. There is significant allele association of SNP rs1801160 with colorectal cancer $(p=0.003$, $\mathrm{OR}=3.264,95 \% \mathrm{CI}=1.425-7.475)$ in present analysis. Haplotype analysis of four DPYD polymorphisms showed significant difference in the distribution „IISt“ haplotype between cases and controls. In comparison to the most common haplotype (VISt), the „IISt" haplotype was associated with increased risk for CRC ( $p=0.038, \mathrm{OR}=2.733,95 \% \mathrm{CI}=1.019-7.326)$. The present study suggests that the SNP rs1801160 and the "IISt" haplotype in the DPYD gene may also have a role in colorectal cancer risk.
\end{abstract}

Key words: Colorectal cancer risk — Dihydropyrimidine dehydrogenase - Genotype - Haplotype analyses

\begin{abstract}
Abbreviations: $\mathrm{CI}$, confidence interval; CRC, colorectal cancer; DPD, dihydropyrimidine dehydrogenase enzyme; $D P Y D$, dihydropyrimidine dehydrogenase gene; EM, expectation-maximization; 5-FU, 5-fluorouracil; OR, odds ratio; SNP, single nucleotide polymorphism.
\end{abstract}

\section{Introduction}

Colorectal cancer (CRC) is one of the most common malignance and the third leading cause of cancer death in world. Recent statistics have indicated that the Slovak Republic has one

Correspondence to: Mária Škereňová, University Hospital in Martin, Department of Clinical Biochemistry, Kollarova 2, 03659 Martin, Slovakia

E-mail:molbiol@mfn.sk of the highest rates of CRC worldwide, and this applies particularly to Slovak males. Slovak male colorectal cancer incidence rates rank 1st in Europe, while female CRC incidence rates rank 5 th in Europe. From the global perspective, CRC incidence and mortality rates have been highest in Central and West European countries. In 2012, Slovakia, Hungary, Denmark, Netherlands, and Czech Republic had the highest incidence rate of CRC among European countries (Ferlay et al. 2013).

Population-based genetic association studies (case-control studies) are the most widely used studies of the impact 
of genetic variants on the risk of developing a particular complex disease. In this way, hundreds of association studies have been performed in order to elucidate the genetic contribution to complex diseases, such as cancer. These studies identified several low penetrance genes that behave as cancer risk modifiers, contributing to the understanding of tumor formation in many types of cancers and leading to advances in diagnosis and therapy.

Dihydropyrimidine dehydrogenase (DPD) is a regulatory enzyme in the pyrimidines catabolic pathway responsible for conversion of thymine or uracil to 5,6-dihydrothymine or 5,6-dihydrouracil, respectively. These intermediates are further metabolized to final products, $\beta$-alanine or $\beta$-aminoisobutyric acid, respectively. $\beta$-aminoisobutyric acid act as a rough indicator for rate of DNA turnover. DPD activity varies in human tissues. Its activity is especially high in hepatic cells and in peripheral blood mononuclear cells. Among Caucasian populations partial deficiency of DPD activity affects between $1 \%$ to $7 \%$ of people and its total loss is very rare, affecting about $0.2 \%$ of people. DPD deficiency is diagnosed by the accumulation of uracil and thymine and results in excess quantities of these molecules in the blood, urine, and cerebrospinal fluid (van Gennip and van Kuilenburg 2000; Maring et al. 2005). The importance and difficulty of clinical recognition is underlined by the fact that pyrimidine defects can be the basis of unexplained anemia (megaloblastic, haemolytic or aplastic), delayed development, epilepsy, neonatal fitting, hyper- or hypotonicity, microcephaly, mental retardation, dysmorphic features, neurogastrointestinal symptoms, ophthalmoplegia, malabsorption, muscle atrophy, polyneuropathy, and toxic reactions to pyrimidine analogue drugs (van Gennip and van Kuilenburg 2000).

Human dihydropyrimidine dehydrogenase (DPYD) gene is located on chromosome 1p21.3, encompassing 23 exons and spanning approximately $843 \mathrm{~kb}$. More than 50 mutations in the DPYD gene have been identified and these mutations interfere with the failure of uracil and thymine metabolism. Genetic variations of DPYD can cause an enzyme deficiency, which could result in severe toxicity or other adverse side effects such as DNA or RNA damages caused by an imbalance of the nucleotide pool. Differences between various population groups as well as between individuals are expected. The incidence of DPD deficiency is reported to be $2.7 \%$ in patients with cancer, but partial DPD deficiency is more common affecting $12.3 \%$ of black women, $4.0 \%$ of black men, $3.5 \%$ of white women and $1.9 \%$ of white men (Saif 2013).

Mutations in the DPYD gene have a dramatic effect on chemotherapy with drugs structurally similar to the pyrimidines (5-fluorouracil (5-FU), capecitabine). DPD deactivates more than $80 \%$ of standard doses of 5 -FU. Some studies reported that DPD had the predictive value on sensitivity to 5-FU and prognostic value on long-term survival (Van
Kuilenburg et al. 1999, 2001, 2002; Ciccolini et al. 2006; Hammond et al. 2016).

Several DPYD variants (rs3918290, rs72547602, rs55886062, 67376798 ) are associated with risk for 5-FU toxicity, such as capecitabine or tegafur (Ezzeldin et al. 2004; Maring et al. 2005; Baskin et al. 2013).

The aim of the present study was to investigate whether the distribution of the DPYD genotypes and haplotypes are over-represented in patients with sporadic CRC compare to healthy controls, and to establish whether these genotypes and haplotypes can be as a risk factor for CRC development in Slovak population.

\section{Materials and Methods}

\section{Study subjects}

A total of 273 patients with sporadic CRC were included in the study. The control group had 187 healthy unrelated volunteers. All subjects were Caucasians of European origin. Healthy donors had negative both past medical and family histories of CRC. Informed consent was obtained from all study subjects. This study was approved by the Human Subjects Committees at Jessenius Faculty in Medicine in Martin, Comenius University in Bratislava. All procedures performed in studies involving human participants were in accordance with the 1964 Helsinki declaration and its later amendments or comparable ethical standards.

\section{DNA analysis}

Genomic DNA was isolated from peripheral blood leukocytes by SiMaxTM Genomic DNA Extraction kit (Cat. No. CSC-50, SBS Genetech Co., Ltd., Beijing, China) according to the manufacturer's instructions. The dsDNA quantitation was conducted on common laboratory spectrophotometer according standard protocol (Barbas 2007). DNA concentration was adjusted to $30 \mu \mathrm{g} / \mathrm{ml}$ and DNA was stored at $-20^{\circ} \mathrm{C}$ for further analysis. All genotyping analyses were performed using high-resolution melting analysis (HRMA) on LightCycler ${ }^{\circledast} 480$ II (Roche) using LightCycler ${ }^{\circledast} 480$ High Resolution Melting Master (Cat. No. 04909631 001, Roche Diagnostics GmbH, Mannheim, Germany) according to the manufacturer's instructions. Designing of all required primer sequences (Table 1) was performed by Primer3Plus software (Untergasser 2012). Conditions for all seven PCR reactions were as follows: initial denaturation at $95^{\circ} \mathrm{C}$ for 5 minutes, annealing temperature $58^{\circ} \mathrm{C}$ and elongation temperature $72^{\circ} \mathrm{C}$, duration of all termocycling steps were 10 seconds and reactions were carried out in total 45 cycles with final extension $72^{\circ} \mathrm{C}$ for 4 minutes. In PCR reactions with $15 \mu$ of reaction volume were the concentrations of primers $0.2 \mu \mathrm{M}$ 
Table 1. HRMA characterization for all input SNPs

\begin{tabular}{|c|c|c|c|c|}
\hline RefSNP primers & Sequence $\left(5^{\prime}-3^{\prime}\right)$ & AA (cDNA) position & $\mathrm{L}(\mathrm{bp})$ & $\mathrm{MI}\left({ }^{\circ} \mathrm{C}\right)$ \\
\hline rs1801267_F & TCTTGTTTTCTGCTATGATTTTCTT & p.Arg886His (c.2657G >A) & 59 & $70-90$ \\
\hline rs1801267_R & ACTGCCAAGTTTTGGACCTT & & & \\
\hline rs72547602_F & CCTGTACAAGTGTCGGTTATGG & p.Asp974Val (c.2921A>T) & 75 & $70-90$ \\
\hline rs72547602_R & ССTCTATTTCTGTTTGCAGGCTA & & & \\
\hline rs3918290_F & TCACCAACTTATGCCAATTCTC & c. $1905+1 G>A$ & 70 & $65-85$ \\
\hline rs3918290_R & AAGGCTGACTTTCCAGACAA & & & \\
\hline rs1801158_F & CAATCCGGCCATTTCTACA & p.Ser534Asn (c.1601G $>A)$ & 75 & $70-90$ \\
\hline rs1801158_R & TCTGCCAAGCCTGAACTACC & & & \\
\hline rs1801160_F & CCCATCAGACCTGAGACA & p.Val732Ile (c.2194G>A) & 63 & $75-95$ \\
\hline rs1801160_R & TGTGTCTTGCATAGGTGGT & & & \\
\hline rs1801159_F & CGAATCATTGATGTGCTGGT & p.Ile543Val (c.1627A>G) & 76 & $70-90$ \\
\hline rs1801159_R & AAATGGCCGGATTGAAGTTT & & & \\
\hline rs1801265_F & TAATTTCTTGGCCGAAGTG & p.Cys29= $($ c. $85 \mathrm{C}>\mathrm{T})$ & 54 & $70-90$ \\
\hline rs1801265_R & AATCCTCGAACACAAACTCA & & & \\
\hline
\end{tabular}

AA, amino acid; cDNA, coding DNA; L, length of amplicons; MI, melting temperature range.

and of magnesium ions $3 \mathrm{mM}$. Amplifications were followed by high resolution melting analysis (HRMA) in temperature range $60-95^{\circ} \mathrm{C}$. Lengths of amplicons and melting intervals are also shown in Table 1. In all reactions, positive and negative controls were included. As a quality control, about $10 \%$ of all samples were repeated as blinded duplicates.

\section{DPYD gene analysis}

Seven single nucleotide polymorphisms (SNPs) of the DPYD gene, all except one within coding region, were genotyped. The splice site mutation rs3918290 localized in intron 14 is associated with rare recessive disorder dihydropyrimidine dehydrogenase deficiency (DPD) and we included it in the study (Van Kuilenburg et al. 1999). The basic characteristics of analyzed SNPs are summarized in Table 1 with further details described elsewhere (Sherry et al. 2001; Cunningham et al. 2015).

\section{Statistics}

For descriptive statistics and testing hypothesis SPSS 16 was used (SPSS Inc. Released 2007; SPSS for Windows, Version 16.0. Chicago, SPSS Inc.). Nonparametric Mann-Whitney test was used for testing the differences of age values between control and patient groups. Pearson's $\chi^{2}$ test for contingency tables was used to test distribution of sex between control and patient groups.

Single marker analysis and haplotype analysis were done using SNP \& Variation Suite v8.3 (Golden Helix, Inc., Bozeman, MT, www.goldenhelix.com). The Fisher's exact test was used to estimate a significance of deviation from Hardy-Weinberg equilibrium and to execute the basic allelic association. Pearson's $\chi^{2}$ test for contingency tables was used to examine haplotype associations. A haplotype frequency was estimated using expectation-maximization (EM) algorithm. Adjusted association tests were performed by logistic regression with case/control status as the dependent variable, and age and sex as confounding variable in additive genetic model. For predictive modelling, stepwise format of logistic regression was used. $p$ values less than 0.05 were considered statistically significant. Odds ratios (ORs) with 95\% confidence intervals (CI) were used to assess genetic effect.

\section{Results}

The average age was of $65.18 \pm 11.41$ years in patient group and $45.60 \pm 13.91$ years in control group. There is statistical significant difference in age between studied groups $\left(Z_{\text {adj }}=12.884 ; p<5 \cdot 10^{-6}\right.$ Mann-Whitney test $)$. There is statistical significant difference in representation women between CRC patients and healthy controls $(41.0 \%$ and $69.0 \%$, respectively; $\left.\chi^{2}=34.779, p<5 \cdot 10^{-6}\right)$. The adjustment for two variables (age and gender) was done in patient and healthy control to eliminate incorrect modeling association of results.

\section{Allele associations}

There are two mutations (rs1801267, rs72547602) present in NCBI dbSNP (build 139) that were not detected in our study. The only two CRC patients have heterozygous genotype for rs3918290, all other patients and controls have wild-type homozygous genotype for rs3918290. There is significant allele association of $\mathrm{rs} 1801160$ with CRC $(p=0.003, \mathrm{OR}=3.264$, $95 \% \mathrm{CI}=1.425-7.475)$ in present analysis (Table 2 ). 
Table 2. Allele association analysis for five SNPs of the DPYD gene

\begin{tabular}{|c|c|c|c|c|c|c|}
\hline \multirow{2}{*}{ Marker } & \multirow{2}{*}{$\begin{array}{l}\text { Minor } \\
\text { Allele }\end{array}$} & \multicolumn{2}{|c|}{ Frequency } & \multirow{2}{*}{$p$} & \multirow{2}{*}{ OR } & \multirow{2}{*}{ CI (95\%) } \\
\hline & & Case (273) & Controls (187) & & & \\
\hline rs1801160 & I & 0.059 & 0.019 & 0.003 & 3.264 & $1.425-7.475$ \\
\hline rs3918290 & a & 0.004 & 0.000 & 0.517 & - & - \\
\hline rs1801159 & V & 0.176 & 0.179 & 0.930 & 0.978 & $0.692-1.378$ \\
\hline rs1801158 & $\mathrm{N}$ & 0.020 & 0.005 & 0.086 & 3.824 & $0.842-17.35$ \\
\hline rs1801265 & $\mathrm{c}$ & 0.256 & 0.241 & 0.642 & 1.088 & $0.801-1.476$ \\
\hline
\end{tabular}

Alleles were labeled using one letter amino acid code for missense SNPs in coding region of DPYD gene and nucleotide marks for other. p, Fisher's Exact test; OR, odds ratio; CI, confidence interval.

Table 3. Model association analysis for five SNPs of the DPYD gene (logistic regression adjusted on sex and age)

\begin{tabular}{|c|c|c|c|c|c|c|}
\hline \multirow{2}{*}{ Marker } & \multicolumn{3}{|c|}{ Additive model } & \multicolumn{3}{|c|}{ Dominant model } \\
\hline & $p$ & Odds Ratio & CI $(95 \%)$ & $p$ & Odds Ratio & CI (95\%) \\
\hline rs1801160 & 0.003 & 4.202 & $1.489-11.85$ & 0.003 & 4.301 & $1.506-12.27$ \\
\hline rs3918290 & 0.167 & - & - & 0.167 & - & - \\
\hline rs 1801159 & 0.375 & 1.243 & $0.765-2.018$ & 0.358 & 1.286 & $0.750-2.205$ \\
\hline rs 1801158 & 0.710 & 1.420 & $0.208-9.683$ & 0.739 & 1.390 & $0.189-10.13$ \\
\hline rs1801265 & 0.907 & 0.977 & $0.657-1.450$ & 0.842 & 0.950 & $0.573-1.572$ \\
\hline
\end{tabular}

p, Full vs. Reduced Model test; CI, confidence interval.

Table 4. Haplotype association analysis

\begin{tabular}{llllll}
\hline & \multicolumn{2}{c}{ Frequency } & & & \\
\cline { 2 - 4 } & Cases & Controls & & & \\
& & & & & \\
\hline H4 & & & & \\
\hline VISt & 0.541 & 0.592 & 0.243 & 0.852 & $0.651-1.115$ \\
VISc & 0.214 & 0.208 & 0.690 & 1.068 & $0.773-1.476$ \\
VVSt & 0.133 & 0.147 & 0.630 & 0.911 & $0.624-1.331$ \\
VVSc & 0.031 & 0.029 & 0.869 & 1.067 & $0.492-2.315$ \\
IISt & 0.035 & 0.014 & 0.038 & 2.733 & $1.019-7.326$ \\
VINt & 0.019 & 0.005 & 0.066 & 3.763 & $0.826-17.15$ \\
\hline H2 & & & & & \\
\hline VS & 0.922 & 0.976 & 0.001 & 0.295 & $0.142-0.614$ \\
IS & 0.058 & 0.019 & 0.004 & 3.217 & $1.403-7.376$ \\
VN & 0.019 & 0.005 & 0.073 & 3.664 & $0.803-16.71$ \\
\hline
\end{tabular}

$p, \chi^{2}$ test; H4, order of SNPs in haplotype: rs1801160, rs1801159, rs1801158, rs1801265; H2, order of SNPs in haplotype: rs1801160, rs1801158, SNPs are ordered according to upward position on the reference chromosome. CI, confidence interval.

Table 5. Haplotype trend regression analysis for “ $\mathrm{H} 4$ ” and „H2“ haplotype

\begin{tabular}{lcccc}
\hline & Regressor & $p$ & Odds Ratio & CI (95\%) \\
\hline $\mathrm{H} 4$ & IISt & 0.055 & 12.31 & $0.767-197.5$ \\
$\mathrm{H} 2$ & IS & 0.003 & 17.66 & $2.216-140.6$ \\
\hline
\end{tabular}

$\mathrm{CI}$, confidence interval.
Genotype associations

Under additive and dominant model, after adjusting for age and sex, there is significant genotype association of rs1801160 with CRC $(p=0.003, \mathrm{OR}=4.202,95 \%$ $\mathrm{CI}=1.489-11.854 ; p=0.003, \mathrm{OR}=4.301,95 \% \mathrm{CI}=1.506-$ 12.27 respectively), too (Table 3 ). Adjusted forward stepwise logistic regression, performed to found out the best predictive association model, confirmed the rs 1801160 out of five SNPs as the predictive marker/regressor.

\section{Haplotype associations}

Six haplotypes with frequency more than $1 \%$ was estimated from four SNPs (in order rs1801160, rs1801159, rs1801158, rs1801265) (Table 4). The minor „IISt“ haplotype with frequency only $1.4 \%$ in healthy controls shown significant higher risk for occurrence in CRC patients $(p=0.038, \mathrm{OR}=2.733$, $95 \%$ CI $=1.019-7.326$ ) (Table 4 ). The reduced haplotypes estimated from two SNPs (in order rs1801160, rs1801158) in linkage disequilibrium have one major haplotype „VS“ with frequency more than $97 \%$ and significantly higher occurrence in healthy controls $(p=0.001, \mathrm{OR}=0.295,95 \%$ $\mathrm{CI}=0.142-0.614)$. The minor haplotype „IS“ has shown significantly higher risk for occurrence in CRC patients $(\mathrm{p}=0.004$, $\mathrm{OR}=3.217,95 \% \mathrm{CI}=1.403-7.376$ ) (Table 4). Haplotype trend regression, stepwise with forward selection and adjusted for 
sex and age, yielded statistically significant results for model with case-control status as independent variable, gender and age as covariates, and only „IS“ haplotype as regressor $(p=0.003, \mathrm{OR}=17.656,95 \% \mathrm{CI}=2.216-140.6)$ (Table 5).

\section{Discussion}

Genetic polymorphisms of pyrimidine metabolism enzymes have been reported to be associated with risk of cancer. It could be hypothesized that there is an association between the gene polymorphisms in DPYD and cancer risk, based on a few reports which have been published (Tanaka et al. 2005; Figueiredo et al. 2013; Kelemen et al. 2014).

The results of our study suggested a potential role for inherited DPYD variation in pyrimidine metabolism with risk of colorectal cancer. In the present case-control study seven SNPs DPYD genes were tested. No individuals with variant in two SNPs (rs1801267, rs72547602) were observed in patient group in our study population. Frequency of another two SNPs (rs3918290 and rs1801158) was low (from $0 \%$ to $2 \%$ ) in both control and case groups. Our hypothesis about association between pathogenic variants in DPYD gene and increased risk of CRC was confirmed for rare rs 1801160 variant. More complex information about combined variability of four SNPs of DPYD gene comes from haplotype analysis. Haplotypes are more powerful discriminators than single SNP in disease association studies. Genetic linkage, the non-random association of alleles expressed in linkage disequilibrium (LD), reduces number of haplotypes against allele combinations (Clark 2004). In the studied cohorts, out of 16 theoretically possible, only 6 haplotypes with the occurrence above $1 \%$ were estimated using EM algorithm implemented in SVS 8. Three of them represented common haplotypes occurring in patients with frequency about $90 \%$, another three had the frequency less than 5\%. The rare „IISt” haplotype (1.4\% in controls) comprises significant 2.7 -fold higher risk to CRC. Two SNPs (rs1801160, rs1801158) are in strong $\mathrm{LD}$, so we examined reduced haplotype construct only from this ones. Haplotype importance was confirmed by minor "IS" haplotype significantly predominant in CRC, while major "VS" haplotype significantly prevailed in controls. Minor "IS" haplotype with minor alleles on both, rs1801160 and rs1801158, could be a potential predictive marker for CRC.

Risk factors associated with CRC include familial and hereditary factors and environmental lifestyle - related risk factors and/or their interactions. Growing evidence suggests that an appreciable component of the genetic contribution to 'sporadic' colorectal cancer is due to large number of variants individually having small effects, thereby invoking the common disease-common variant paradigm for this type of cancer (Cheah 2009; Tenesa and Dunlop 2009). Colorectal cancer has been extensively studied in the laboratory and the clinics. Due to available technologies, most of this research has been focused on genetic and epigenetic aspects. Abnormality and DPD deficiency can be identified by different genetic analyses and by determination of DPD levels in the peripheral blood mononuclear cells, respectively. Epigenetic modifications of the DPYD promoter region have been identified as one of the most important regulatory mechanisms of DPD enzymatic activity.

Somatic mutations of DPYD are associated with upregulation of pyrimidine degradation, nucleotide synthesis, and nucleic acid processing while salvage and nucleotide conversion is downregulated in human skin coetaneous melanoma (Lauren et al. 2016). The relative importance of salvage versus de novo synthesis likely depends on the growth conditions and on the tissue specificity. Recently, it has been published that salvage pathways are very important for surviving and proliferation of cancer cells in colorectal cancers (Qi and Voit 2014).

Cancer cells take advantage of distinct metabolic pathways promoting cellular proliferation or oncogenic progression (Boroughs and DeBerardinis 2015).

Lauren et al. (2016) showed a comprehensive view on how a hypermutated metabolic gene deregulates pyrimidine and nucleic acid synthesis and promotes malignant progression of melanoma.

SNPs, deletion mutations, splicing deficits, frameshift mutations and methylation of DPYD gene may be associated with partial or total loss of DPD activity (Johnson et al. 1997; Yu et al. 2006). Based on the complexity of DPYD gene (23 exons), a lot of sequence variations could be associated with cancer progression (Ezzeldin et al. 2003; Ogura et al. 2005; van Kuilenburg et al. 2005). So far, more than 130 genetic polymorphisms have been recorded in NCBI dbSNP in the coding, intronic and untranslated $3^{\prime}$ and 5 ' regions of $D P Y D$. Conditions resulting in a mutant $D P Y D$ allele include base substitutions, splicing deficits and frameshift mutations (Panczyk 2014; Lauren et al. 2015). The most frequently observed variants are rs1801159, rs1801160, and rs1801265 at frequencies of $11.5-30 \%, 0.7-9 \%$, and $2.9-13.7 \%$, respectively, but data regarding to their effects on DPD activity are disputable. Moreover, most $D P Y D$ variants and phenotypic consequence are of very low frequency, and several studies did not find any individuals with these variants (Caudle et al. 2013).

Nowadays, genetic polymorphisms of pyrimidine metabolism enzymes have been reported to be associated with higher risk of cancer, but the physiologic role of this enzyme in cancer cells is still unknown (Amstutz et al. 2011). Shaul et al. (2014) demonstrated that DPD plays an essential role in the epithelial-mesenchymal transition, providing one of the first indications for its function in cancer. However, there is a clear distinction between this function and the 
normal role of DPD in the liver. Recently, the association of $D P Y D$ variants rs 11587873 and rs828054 with modulated risk of ovarian cancer (Kelemen et al. 2014) or malignant lymphoma (Figueiredo et al. 2013) has observed. The study of Tanaka et al. (2005) suggested that influence of DPYD Cys29Arg (rs1801265) posed only a limited risk for the six malignancies in Japanese.

In conclusion, this study suggests that rs 1801160 and the "IISt" haplotype in the DPYD gene may also have a role in colorectal cancer risk in the Slovak population, indicating the importance of this gene involved in pyrimidine metabolism with respect to cancer risk. Further studies of incident case - control populations will be required to confirm this hypothesis. Studies of this association among other ethnic groups also remain to be done.

Acknowledgement. This publication is the result of the project implementation: "CENTER OF TRANSLATIONAL MEDICINE", ITMS: 26220220021 supported by the Operational Programme Research and Innovation funded by the ERDF. This work was supported by the Slovak Research and Development Agency under the contract No. APVV-15-0217.

Conflict of interest. No conflicts of interest are declared.

\section{References}

Amstutz U., Froehlich T. K., Largiadèr C. R. (2011): Dihydropyrimidine dehydrogenase gene as a major predictor of severe 5-fluorouracil toxicity. Pharmacogenomics 12, 13211336 https://doi.org/10.2217/pgs.11.72

Barbas C. F., Burton D. R., Scott J. K., Silverman G. J. (2007) Quantitation of DNA and RNA. CSH Protoc. 2007, pdb.ip47 https://doi.org/10.1101/pdb.ip47

Baskin Y., Amirfallah A., Unal O. U., Calibasi G., Oztop I. (2013): Dihydropyrimidine dehydrogenase $85 \mathrm{~T}>\mathrm{C}$ mutation is associated with ocular toxicity of 5-fluorouracil: A case report. Am. J. Ther. 22, e36-39 https://doi.org/10.1097/MJT.0b013e31829e8516

Boroughs, L. K. and DeBerardinis, R. J. (2015): Metabolic pathways promoting cancer cell survival and growth. Nature Cell Biology 17, 351-359 https://doi.org/10.1038/ncb3124

Caudle, K. E., Thorn, C. F., Klein, T. E., Swen, J. J., McLeod, H. L., Diasio, R. B., Schwab, M. (2013): Clinical Pharmacogenetics Implementation Consortium guidelines for dihydropyrimidine dehydrogenase genotype and fluoropyrimidine dosing. Clin. Pharmacol. Ther. 94, 640-645 https://doi.org/10.1038/clpt.2013.172

Cheah P. Y. (2009): Recent advances in colorectal cancer genetics and diagnostics. Crit. Rev. Oncol. Hematol. 69, 45-55 https://doi.org/10.1016/j.critrevonc.2008.08.001

Ciccolini J., Mercier C., Evrard A., Dahan L., Boyer J. C., Duffaud F., Richard K., Blanquicett C., Milano G., Blesius A. (2006): A rapid and inexpensive method for anticipating severe toxic- ity to fluorouracil and fluorouracil-based chemotherapy. Ther. Drug Monit. 28, 678-685 https://doi.org/10.1097/01.ftd.0000245771.82720.c7

Clark A. G. (2004): The role of haplotypes in candidate gene studies. Genet. Epidemiol. 27, 321-333 https://doi.org/10.1002/gepi.20025

Cunningham F., Amode M. R., Barrell D., Beal K., Billis K., Brent S., Carvalho-Silva D., Clapham P., Coates G., Fitzgerald S. et al. (2015): Ensembl 2015. Nucleic Acids Res. 43, D662-669 https://doi.org/10.1093/nar/gku1010

Ezzeldin H., Diasio R. (2004): Dihydropyrimidine dehydrogenase deficiency, a pharmacogenetic syndrome associated with potentially life-threatening toxicity following 5 -fluorouracil administration. Clin. Colorectal Cancer 4, 181-189 https://doi.org/10.3816/CCC.2004.n.018

Ezzeldin H., Johnson M. R., Okamoto Y., Diasio R. B. (2003): Denaturing high performance liquid chromatography analysis of the DPYD gene in patients with lethal 5-fluorouracil toxicity. Clin. Cancer Res. 9, 3021-3028

Ferlay J., Soerjomataram I., Ervik M., Dikshit R., Eser S., Mathers C., Rebelo M., Parkin D. M., Forman D., Bray F. (2015): Cancer incidence and mortality worldwide: sources, methods and major patterns in GLOBOCAN 2012. Int. J. Cancer 136, 359-386 https://doi.org/10.1002/ijc.29210

Figueiredo J. C., Levine A. J., Crot J. W., Baurley J., Haile R. W. (2013): Folate-genetics and colorectal neoplasia: what we know and need to know next. Mol. Nutr. Food Res. 57, 607-627 https://doi.org/10.1002/mnfr.201200278

Hammond W. A., Swaika A., Mody K. (2016): Pharmacologic resistance in colorectal cancer: a review. Ther. Adv. Med. Oncol. 8, 57-84 https://doi.org/10.1177/1758834015614530

Johnson M. R., Wang K., Tillmanns S., Albin N., Diasio R. B. (1997): Structural organization of the human dihydropyrimidine dehydrogenase gene. Cancer Res. 57, 1660-1663

Kelemen, L. E., Terry, K. L., Goodman, M. T., Webb, P. M., Bandera, E. V., McGuire, V. et al. (2014): Consortium analysis of gene and gene-folate interactions in purine and pyrimidine metabolism pathways with ovarian carcinoma risk. Mol. Nutr. Food Res. 58, 2023-2035

https://doi.org/10.1002/mnfr.201400068

Lauren E., Rohit G., Fabian V. F. (2016): Hypermutation of DPYD deregulates pyrimidine metabolism and promotes malignant progression. Mol. Cancer Res. 14, 196-206 https://doi.org/10.1158/1541-7786.MCR-15-0403

Maring J. G. , Groen H. J. M., Wachters F. M., Uges D. R. A., de Vries E. G. E. (2005): Genetic factors influencing pyrimidineantagonist chemotherapy. Pharmacogenomics J. 5, 226-243 https://doi.org/10.1038/sj.tpj.6500320

Ogura K., Ohnuma T., Minamide Y., Mizuno A., Nishiyama T., Nagashima S. (2005): Dihydropyrimidine dehydrogenase activity in 150 healthy Japanese volunteers and identification of novel mutations. Clin. Cancer Res. 11, 5104-5111 https://doi.org/10.1158/1078-0432.CCR-05-0217

Panczyk M. (2014): Pharmacogenetics research on chemotherapy resistance in colorectal cancer over the last 20 years. World J. Gastroenterol. 20, 9775-9827 https://doi.org/10.3748/wjg.v20.i29.9775 
Qi Z., Voit E. O. (2014): Identification of cancer mechanisms through computational systems modeling. Transl. Cancer Res. 3, 233-242 http://doi.org/10.3978/j.issn.2218-676X.2014.05.03

Saif M. W. (2013): Dihydropyrimidine dehydrogenase gene (DPYD) polymorphism among Caucasian and non-Caucasian patients with 5-FU- and capecitabine-related toxicity using full sequencing of DPYD. Cancer Genomic Proteomics 10, 89-92

Shaul Y. D., Freinkman E., Comb W. C., Cantor J. R., Tam W. L., Thiru P., Kim D., Kanarek N., Pacold M. E., Chen W. W. et al. (2014): Dihydropyrimidine accumulation is required for the epithelial-mesenchymal transition. Cell 158, 1094-1109 https://doi.org/10.1016/j.cell.2014.07.032

Sherry S. T., Ward M. H., Kholodov M., Baker J., Phan L., Smigielski E. M., Sirotkin K. (2001): dbSNP: the NCBI database of genetic variation. Nucleic Acids Res. 29, 308-311 https://doi.org/10.1093/nar/29.1.308

Tanaka D., Hishida A., Matsuo K., Iwata H., Shinoda M., Yamamura Y., Kato T., Hatooka S., Mitsudomi T., Kagami Y. et al. (2005): Polymorphism of dihydropyrimidine dehydrogenase (DPYD) Cys29Arg and risk of six malignancies in Japanese. Nagoya J. Med. Sci. 67, 117-124

Tenesa A., Dunlop M. G. (2009): New insights into the aetiology of colorectal cancer from genome-wide association studies. Nat. Rev. Genet. 10, 353-358 https://doi.org/10.1038/nrg2574

Untergasser A., Cutcutache I., Koressaar T., Ye J., Faircloth B. C., Remm M., Rozen S. G. (2012): Primer3-new capabilities and interfaces. Nucleic Acids Res. 40, e115 https://doi.org/10.1093/nar/gks596

Van Gennip A. H., Van Kuilenburg A. B. (2000): Defects of pyrimidine degradation: clinical, molecular and diagnostic aspects. Adv. Exp. Med. Biol. 486, 233-241 https://doi.org/10.1007/0-306-46843-3_46

Van Kuilenburg A. B. P., Meinsma R., Beke E., Bobba B., Boffi P., Enns G. M. (2005): Identification of three novel mutations in the dihydropyrimidine dehydrogenase gene associated with altered pre-mRNA splicing or protein function. Biol. Chem. 386, 319-324

https://doi.org/10.1515/BC.2005.038

Van Kuilenburg A. B., Meinsma R., Zoetekouw L., Van Gennip A. H. (2002): High prevalence of the IVS14 +1G>Amutation in the dihydropyrimidine dehydrogenase gene of patients with severe 5-fluorouracil-associated toxicity. Pharmacogenetics 12, 555-558 https://doi.org/10.1097/00008571-200210000-00007

Van Kuilenburg A. B., Muller E. W., Haasjes J., Meinsma R., Zoetekouw L., Waterham H. R., Baas F., Richel D. J., Van Gennip A. H. (2001): Lethal outcome of a patient with a complete dihydropyrimidine dehydrogenase (DPD) deficiency after administration of 5-fluorouracil: frequency of the common IVS14+1G>Amutation causing DPD deficiency. Clin. Cancer Res. 7, 1149-1153

Van Kuilenburg A. B., Vreken P., Abeling N. G., Bakker H. D., Meinsma R., Van Lenthe H., De Abreu R. A., Smeitink J. A., Kayserili H., Apak M. Y. et al. (1999): Genotype and phenotype in patients with dihydropyrimidine dehydrogenase deficiency. Hum. Genet. 104, 1-9 https://doi.org/10.1007/PL00008711

Yu J., McLeod H. L., Ezzeldin H. H., Diasio R. B. (2006): Methylation of the DPYD promoter and dihydropyrimidine dehydrogenase deficiency. Clin. Cancer Res. 12, 3864 https://doi.org/10.1158/1078-0432.CCR-06-0549

Received: April 11, 2017

Final version accepted: October 10, 2017 